\title{
REFLEX TENSION DURING SINUSOIDAL MOVEMENT IN GASTROCNEMIUS AND SOLEUS OF DECEREBRATED CAT*
}

\author{
Yasuhiko TamaI,** Richard Herman, and William Freedman \\ Department of Rehabilitative Medicine, Temple University School of Medicine, \\ and \\ Krusen Research Center, Moss Rehabilitation Hospital, Philadelphia, Pennsylvania
}

Summary 1. Reflex tension, electromyograph (EMG) and afferent discharges were observed during sinusoidal movement in the gastrocnemius and soleus muscles of decerebrated cat.

2. The amplitude of tension began to decrease at over $9 \mathrm{~Hz}$ in the gastrocnemius and at over $5 \mathrm{~Hz}$ in the soleus. The phase angle of reflex tension showed the phase lead at low frequencies and the phase lag at over $14 \mathrm{~Hz}$ in the gastrocnemius and at over $7 \mathrm{~Hz}$ in the soleus. The alternate cycle fluctuation of tension was observed at over $22 \mathrm{~Hz}$ in the gastrocnemius and at over $14 \mathrm{~Hz}$ in the soleus.

3. The phase lead of EMG was amplified with increasing frequency but began to recede at over $8 \mathrm{~Hz}$ in the gastrocnemius and at over $4 \mathrm{~Hz}$ in the soleus, while the amplitude of EMG increased. The alternate fluctuation of EMG was seen at the same frequency as the alternate cycle fluctuation of tension.

4. The effect of reflex tension on stretch receptors was investigated by simultaneous recording of dorsal rootlet GIa and GIb discharges, reflex tension and EMG. The GIa discharges increased with sinusoidal movement but partial acceleration and pause of the discharges were observed during a cycle. EMG group discharges were seen after the acceleration and during the GIa discharge pause. GIb discharge pause was not seen during a cycle but discharge acceleration was observed after EMG group discharges.

5. The shift in the GIb discharge possibly occurring as a result of its own reflex tension suggests that it acts as an inhibitor to successive GIa discharges and may be the cause of the alternate cycle fluctuation of the EMG at high frequencies.

Received for publication July 20, 1973

* This paper partially fulfilled the $\mathrm{Ph}$. D. dissertation requirement of Yasuhiko Tamai.

** Present address: Department of Physiology, Wakayama Medical College, Wakayama, Japan

玉井靖彦 
6. These results were discussed by introducing the reflex system time lag due to the conduction time of the nerve and the contraction time of the muscle.

Responses of reflex tension as a final output of the stretch reflex system depend on many factors such as pattern of receptor and motoneuron discharges, time delay in neural circuit and inherent properties of muscle itself. Beginning with the original study by LidDell and SHERrington (1924), these factors in the stretch reflex system of a decerebrate cat have also been studied under static conditions (Granit, 1958) and during slow movement (MATTHEWs, 1959a, b). The dynamic properties of stretch reflex were investigated in the extensor muscles of the cat (LipPold et al., 1958; Poppele and Terzuolo, 1968; PARTRIDGe and GlASER, 1960; Roberts, 1963; JANSEN and RACK, 1966; ROSENTHAL et al., 1970) and in various muscles in man (GotTlieb et al., 1970; Herman et al., 1973). In several of these investigations, controlled sinusoidal movement at various frequencies was used to test the response of stretch reflex.

Timing of the reflex tension during sinusoidal stretching was investigated by JANSEN and RACK (1966) who found that it depended on how far the angular advance of EMG combined with the damping properties of muscle offset the time delay in the reflex. Further, Herman et al. (1973) showed that decay in gain and phase lag of tension were altered by the rheologic and contractile properties of sinusoidal-stretched muscle and that a near flat gain and constant phase lead of tension was not the result of compensation for the responses of neural and non-neural systems as was indicated by Poppele and Terzuolo (1968). The inherent properties of muscle contraction, therefore, seem to be important for reflex tension response.

This study reports the application of controlled sinusoidal movement to the gastrocnemius and soleus muscles of a decerebrate cat and describes the change in reflex responses during sinusoidal movement. Comparison of amplitude and phase angle of tension versus frequency curves in the gastrocnemius and soleus muscles demonstrated that the appearance of tension decay, phase lag and alternate cycle fluctuation of tension and EMG occurred at different frequencies. These results will be treated according to their differences in contraction times which correspond to one of the inherent contractile properties of muscle.

\section{METHODS}

Forty-five cats between 3 to $4 \mathrm{~kg}$ in weight were decerebrated at intercollicular level under ether anesthesia after common carotid ligation. The hind limb and hip were denervated, as far as possible, except for the gastrocnemius and soleus. The femur and calcaneus bone were rigidly fixed by pins on a stereotaxic apparatus. The Achilles tendon was clamped directly to an isometric tension transducer 
which was connected to a vibrator in series. Laminectomy was performed between S1 to L5 for the preparations used in recording afferent discharge. The dorsal rootlets of L7 were separated partially in paraffin oil until functionally isolated single filament originating from the GIa and GIb fibers of the gastrocnemius and soleus were obtained. Temperature of the cat was kept between $36^{\circ}$ to $38^{\circ} \mathrm{C}$ by a warm water blanket.

The length of the gastrocnemius was set by elongating the muscle 4 or $8 \mathrm{~mm}$ beyond the point at which the muscle alone produced $50 \mathrm{~g}$ of reflex tension. In case of the soleus, the muscle length was specified as the same as that of its paired gastrocnemius, in consideration of its functional relation in situ. Experiments were delayed until at least three hours after decerebration.

Mechanical Arrangement. Sinusoidal movement was performed by a vibrator (MB Electric Co., model N 229) and controlled by a velocity feedback circuit (specially designed). The vibrator was capable of 2-mm, peak to peak sinusoidal oscillation at any examined frequency. Preparations showing stretch displacement errors greater than $5 \%$ under load were omitted. Each series of vibrations were applied at $30 \mathrm{sec}$ interval. A tension transducer (Statham transducing cell, model UC 3 and load cell accessory, model UL 4) and a displacement transducer (linear potentiometer) were connected to the vibrator in series and used for recording myograms and oscillation displays, respectively. The outputs were recorded on a visicorder (Honeywell, model 5600) via DC amplifiers (Teca, model A-8).

Electrical Arrangement. Silver electrodes were used for recording afferent discharges and wire electrodes (Karma alloy wire, 0.001 inch) with tips stripped about $2 \mathrm{~mm}$ were used for EMG record. One wire electrode was applied to the belly of the gastrocnemius or soleus and the other electrode was placed close to the Achilles tendon. Afferent discharges and EMG were also recorded on the visicorder and FM tape recorder by means of AC amplifiers.

Display of Results. Post-experimentally, a reset mode integrator was used to measure the amplitude and phase angle of the EMG in each cycle. Twenty reset numbers were selected arbitrarily for all frequencies. The integrated EMG of the reset mode was displayed along with the tension and the displacement (see the lowest traces at $0.5,6$, and $12 \mathrm{~Hz}$ in the soleus in Fig. 1). To determine an amplitude and a phase angle of integrated EMG, the height of each integrated EMG was measured and a two-point sliding average was performed between the height of the adjacent integrated EMGs. Initiation of EMG for calculation of a response time was also determined by using the integrated EMG because the beginning of the EMG on the raw data was sometimes hard to determine at low frequencies where there were many small continuous EMG. Thus, the point at which the height of integrated EMG reached $5 \%$ of its total value for each cycle was selected as the initiation of EMG.

Tension amplitude was measured from the valley to the peak of the tension 


\section{GASTROCNEMIUS MUSCLE}

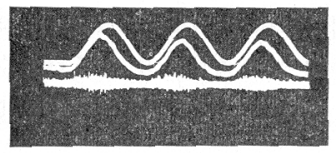

$2 \mathrm{~Hz}$

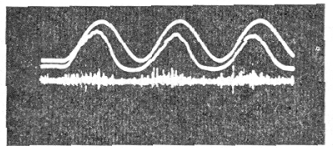

$4 \mathrm{~Hz}$

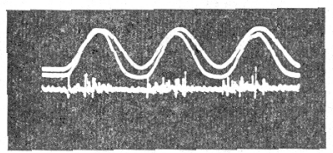

$6 \mathrm{~Hz}$

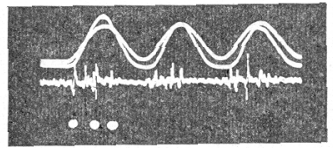

$8 \mathrm{~Hz}$

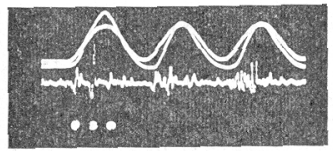

$10 \mathrm{~Hz}$

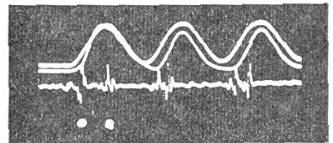

$12 \mathrm{~Hz}$

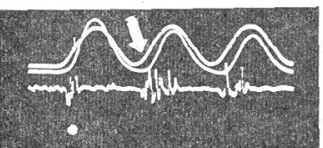

SOLFUS MUSCLE
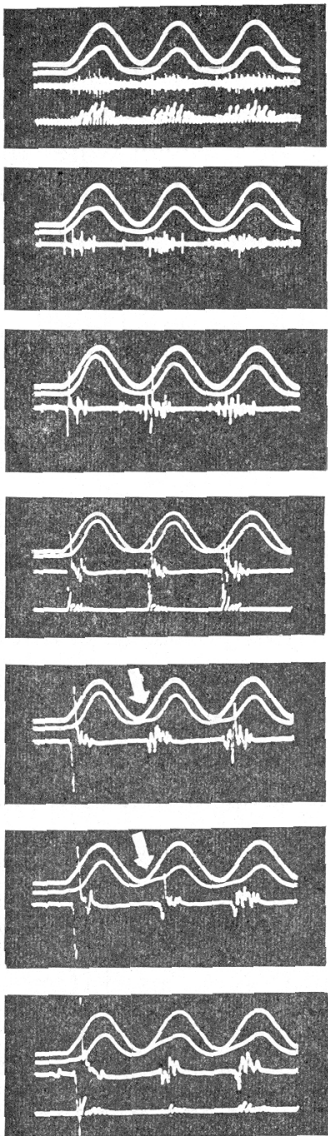

Fig. 1. Reflex tension and EMG during sinusoidal movement (amplitude of displacement: $2 \mathrm{~mm}$ peak to peak, muscle lengthening: $8 \mathrm{~mm}$ ). Traces at each picture show displacement, tension and EMG in that order. The lowest traces at $0.5,6$, and $12 \mathrm{~Hz}$ in the soleus are samples of reset integrated EMG described in the method. Note phase shift of tension among frequencies.

curve, and the phase angle was calculated from the distances between the peak of tension and displacement. The amplitude and phase in the first, third, fifth and seventh were averaged at each frequency in both the tension and EMG. The average amplitude at each frequency was normalized to an average value among preparations at $0.5 \mathrm{~Hz}$ while the phase angle showed the absolute value. 


\section{RESULTS}

\section{Alternate cycle fluctuation of tension and EMG}

Gastrocnemius and soleus muscles of decerebrated cats were stretched sinusoidally for about twenty cycles at various frequencies. As seen from raw data in Fig. 1, the tension curve did not exactly follow the applied sinusoidal movement and showed a sharpened peak and widened valley (peak to peak amplitude of displacement was $2 \mathrm{~mm}$ ). The amplitude of tension during first several cycles were usually larger than the following ones (Higgins et al., 1962). Several other types of fluctuation were noticed in the tension response during the sinusoidal movement.

The fluctuation in the early period of the tension curve (JANSEN and RACK, 1966) was seen at $8 \mathrm{~Hz}$ in the soleus and at $12 \mathrm{~Hz}$ in the gastrocnemius (Fig. 1, 2, arrows). The amplitude of tension in the second cycle became larger than the

\section{GASTROCNEMIUS MUSCLE}

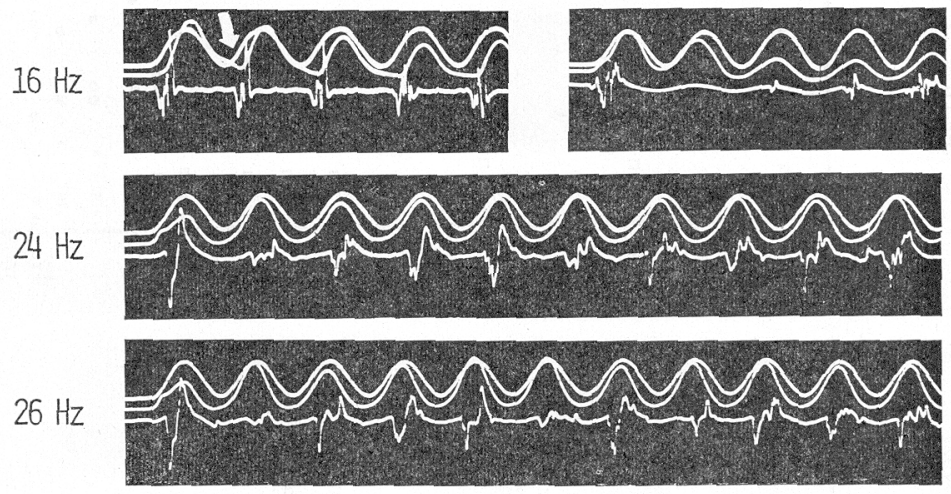

\section{SOLFUS MUSCLE}

$14 \mathrm{~Hz}$

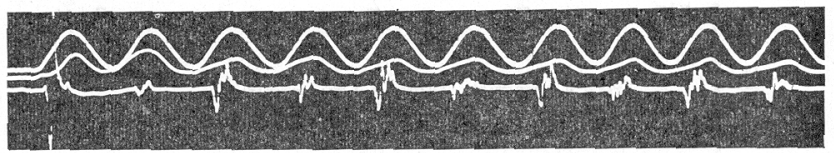

$16 \mathrm{~Hz}$

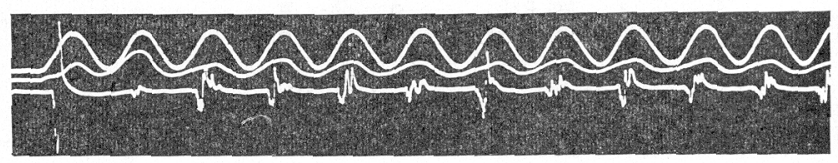

Fig. 2. Reflex tension and EMG during sinusoidal movement at high frequencies (amplitude of displacement: $2 \mathrm{~mm}$ p-p, muscle lengthening: $8 \mathrm{~mm}$ ). Traces show displacement, tension and EMG in that order. Note alternate cycle fluctuation of tension and EMG among frequencies. 
one in the first cycle at 22 or $24 \mathrm{~Hz}$ in the gastrocnemius and at 14 or $16 \mathrm{~Hz}$ in the soleus, despite the obviously smaller EMG response in the second cycle. This relation was seen not only in the first two cycles but also in the following cycles (Fig. 2). An 'alternate cycle fluctuation of tension and EMG' relationship was observed by a depression of EMG usually occurring in the cycle where the tension was larger than the previous one. In a few preparations, the EMG was diminished at around $16 \mathrm{~Hz}$ in the gastrocnemius without accompanying larger tension than previous one (see right picture at $16 \mathrm{~Hz}$ in Fig. 2). At high frequencies over $22 \mathrm{~Hz}$ in the gastrocnemius and at over $16 \mathrm{~Hz}$ in the soleus, the large EMG produced by stretch reflex, therefore, seems to arise from the large tension not in the cycle where the EMG occurs but in the subsequent cycle in which there is a depression of the EMG.
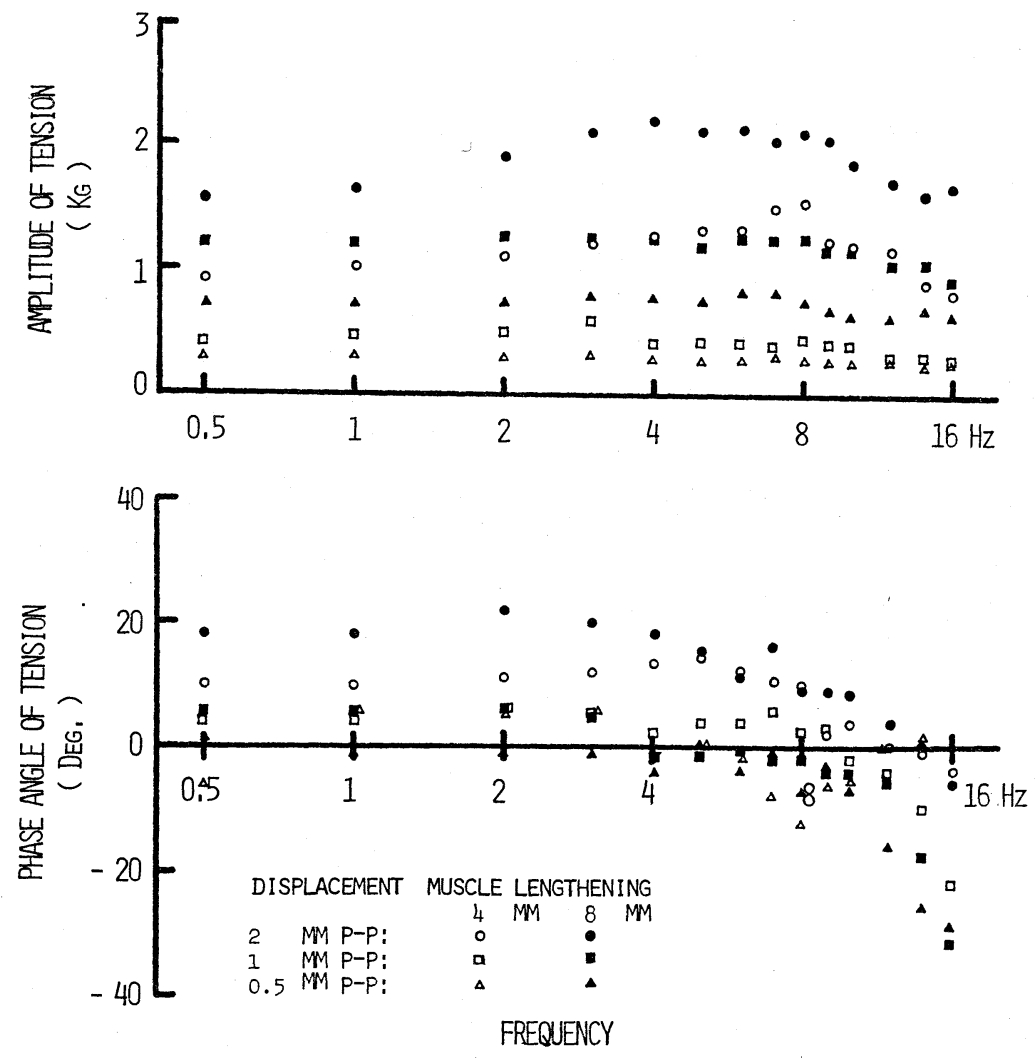

Fig. 3. Amplitude (upper graph) and phase angle (lower graph) of tension versus frequency in the gastrocnemius at various sinusoidal movements. Amplitude of displacement was $2 \mathrm{~mm} \mathrm{p}$-p (circles), $1 \mathrm{~mm} \mathrm{p}$-p (squares) or $0.5 \mathrm{~mm} \mathrm{p}$-p (triangles). Open and closed symbols show $4 \mathrm{~mm}$ and $8 \mathrm{~mm}$ muscle lengthening, respectively. Note tension decrease and phase lag at high frequencies. 
Amplitude and phase angle of tension and EMG

Figure 3 illustrates the amplitude (upper graph) and phase angle (lower graph) of tension versus frequency curve in the gastrocnemius (muscle lengthening: 4 or $8 \mathrm{~mm}$ ) at various amplitudes of sinusoidal movement.

The amplitude of tension increased slightly with increasing frequency, especially in large displacement of sinusoidal movement (closed and open circles), but decreased at high frequencies over about $9 \mathrm{~Hz}$. The peak of tension preceded the peak of displacement (phase lead: + degree) at low frequencies and it occurred after the peak of displacement (phase lag: - degree) at high frequencies. The critical frequency beyond which the tension decay or phase lag occurred was different from preparation to preparation but the tension always decreased at higher frequencies where the phase lag was seen. Tension decrease, however, was not noticed when a small amplitude of sinusoidal movement was applied.

These results pose the following questions: what kind of factor or factors cause the tension decay and phase lag at high frequencies, and are the alternate cycle fluctuation of tension and EMG mentioned in a previous section caused by

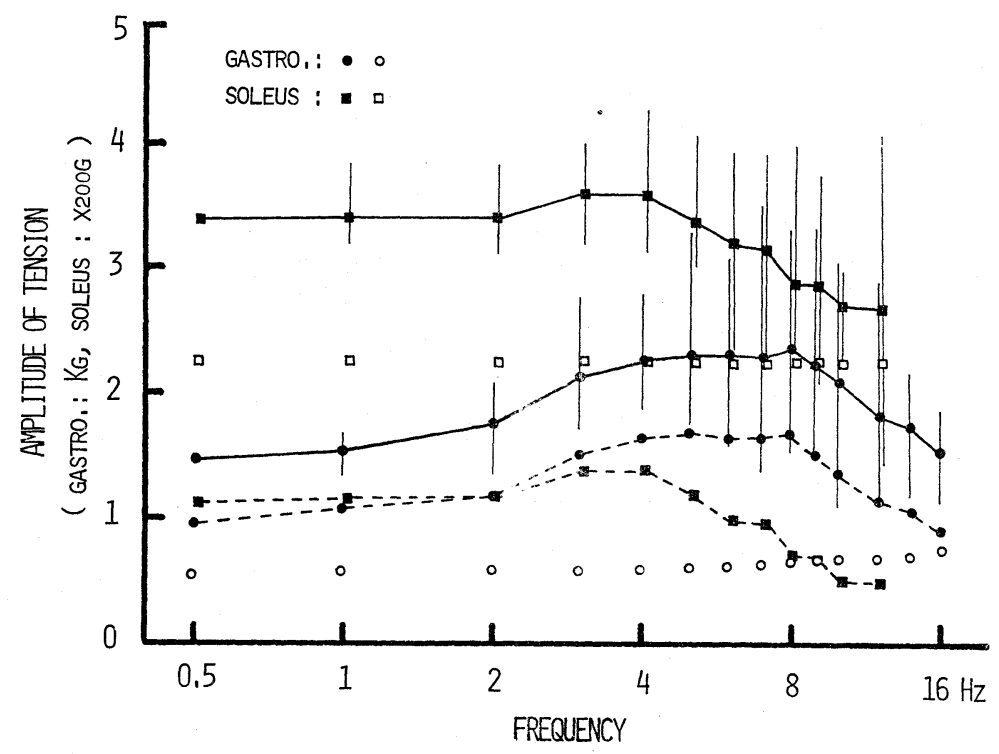

Fig. 4. Amplitude of tension versus frequency during sinusoidal movement ( $2 \mathrm{~mm} \mathrm{p}-\mathrm{p})$ in the gastrocnemius (circle symbols) and in the soleus (square symbols) at $8 \mathrm{~mm}$ muscle lengthening. Tension was normalized to an average value among examined preparations at $0.5 \mathrm{~Hz}$. Vertical lines on the closed symbols show variation among normalized preparations. Open circles and squares indicate average value of normalized passive tension after cutting tibial nerve. Closed circles and squares with broken line show active reflex tension in the gastrocnemius and soleus respectively (closed symbol minus open symbol in the muscle). Decay of tension was observed at over $9 \mathrm{~Hz}$ in the gastrocnemius and at over $5 \mathrm{~Hz}$ in the soleus. 
a similar factor in the stretch reflex? The amplitude and phase angle of tension and EMG in the gastrocnemius were, therefore, compared with those in the soleus using large sinusoidal displacement ( $2 \mathrm{~mm}$ - $\mathrm{p}$ ) which easily produced tension decay and phase lag.

As seen in Fig. 4, the amplitude of tension increased gradually with increasing frequencies up to about $8 \mathrm{~Hz}$ in the gastrocnemius (solid circles with solid line) and up to about $4 \mathrm{~Hz}$ in the soleus (solid squares with solid line). In both muscles, the tension began to decrease at greater frequencies. A similar result has been described in the soleus by JANSEN and RACK (1966). Passive tension due to viscoelastic property of muscle remained almost constant at low frequencies and slightly increased at high frequencies (open circles and squares). Tension increase and decay, therefore, reflect the responses of active tension caused by stretch reflex (closed circle and square on the broken line).

Figure 5 shows the phase angle of tension versus frequency curves in the gastrocnemius (closed circle on the solid line) and soleus (solid square on the solid line). The phase lead of gastrocnemius and soleus were about 18 degrees and 10 degrees at 0.5 to $3 \mathrm{~Hz}$, respectively. As frequency increased, the phase angle of tension curve receded to zero degree and finally the phase lag occurred at $14 \mathrm{~Hz}$ in the gastrocnemius and at around $7 \mathrm{~Hz}$ in the soleus, while the phase angle due

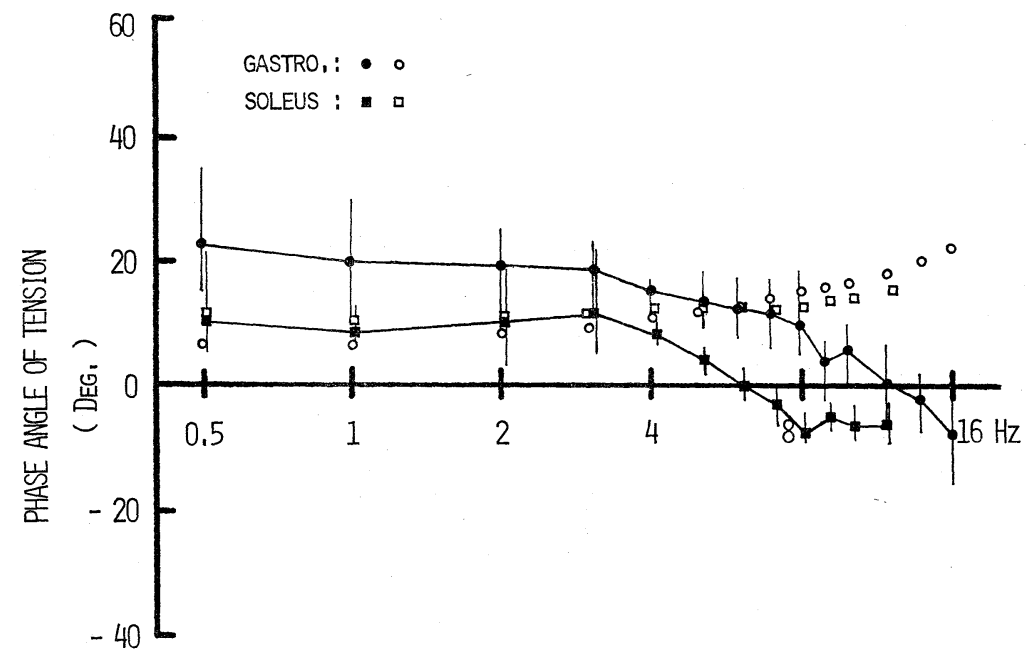

FREQUENCY

Fig. 5. Phase angle of tension versus frequency during sinusoidal movement ( $2 \mathrm{~mm} \mathrm{p}-\mathrm{p})$, in the gastrocnemius (circle symbols) and in the soleus (square symbols) at $8 \mathrm{~mm}$ muscle lengthening. Phase angle of tension indicates absolute value calculated from peak of displacement ( 0 degree) with variation among examined preparations (vertical line). Open circles and squares show average of phase angle of passive tension in the muscles. Note phase lag at over $14 \mathrm{~Hz}$ in the gastrocnemius and at over $7 \mathrm{~Hz}$ in the soleus. 
to viscoelastic properties of these muscle showed the phase lead and increased gradually as applied frequency increased (open circles and squares).

From a comparison of the results, it was noticed that the tension decay, phase lag and alternate cycle fiuctuation of tension and EMG were observed similarly in both muscles but appeared at a different frequency. The frequency which caused these results was always lower in the soleus than in the gastrocnemius.

The EMG response as a measure of motor fiber out was recorded with muscle tension during sinusoidal movement, and the possible influence of EMG on the reflex tension as an input to the muscle was studied. Figure 6 shows the amplitude of EMG versus frequency curves with greater increasing frequencies both in the gastrocnemius (closed circles with solid line) and soleus (closed squares with solid line). There usually was an increase of amplitude at high frequencies,

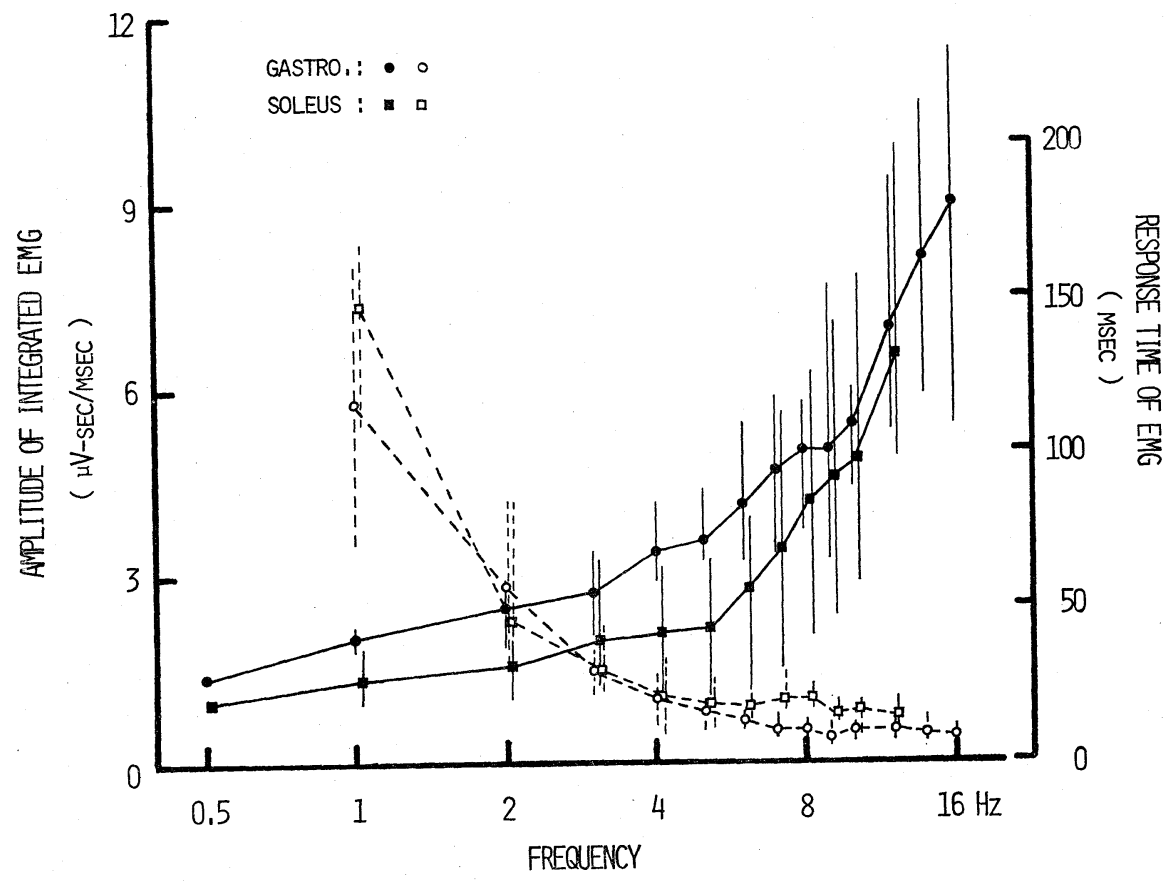

Fig. 6. Amplitude and response time of EMG versus frequency during sinusoidal movement ( $2 \mathrm{~mm} \mathrm{p}-\mathrm{p}$ ) in the gastrocnemius (circle symbols) and in the soleus (square symbols) at $8 \mathrm{~mm}$ muscle lengthening. Amplitude of EMG was normalized to average value among examined preparation at $0.5 \mathrm{~Hz}$ and showed with variation among normarized value (vertical line). Open circles and squares with broken line show average of absolute response time of the first EMG calculated from initiation of sinusoidal movement at each frequency with variation among examined preparations. Amplitude of EMG increased with increasing frequencies and response time became short and close to response time of tendon jerk at over $8 \mathrm{~Hz}$ in both muscles. 
however, the tension showed a decrease. The shape of EMG discharge resembled the response of a tendon jerk at high frequencies. Response times of the first EMG measured from an initiation of sinusoidal movement (open circles and squares with broken line) became short with increasing frequencies and almost similar to that of the tendon jerk (about $8 \mathrm{msec}$ ) in both muscles. Therefore, the large increase of EMG amplitude indicates that motor neurons which supply the muscles examined in this study are activated synchronously by sinusoidal movement at high frequencies.

The EMG curve showed a larger phase lead than the tension curve (Fig. 7). The phase lead was about 40 degrees in the gastrocnemius and 25 degrees in the soleus at low frequencies. As frequency increased, the phase lead of EMG reached about 130 degrees at $7 \mathrm{~Hz}$ in the gastrocnemius and about 120 degrees at $3 \mathrm{~Hz}$ in the soleus but began to decrease beyond these frequencies. The regression of EMG phase angle at high frequencies over $8 \mathrm{~Hz}$ in both muscles paralleled the broken line in Fig. 7. The broken line indicates the hypothetical position of the first EMG which has a constant time lag of $8 \mathrm{msec}$, that is a time delay in the neural circuit. The time lag calculated by the tendon jerk consists of a reaction time of receptor, conduction time of peripheral nerve, synaptic delay and latency

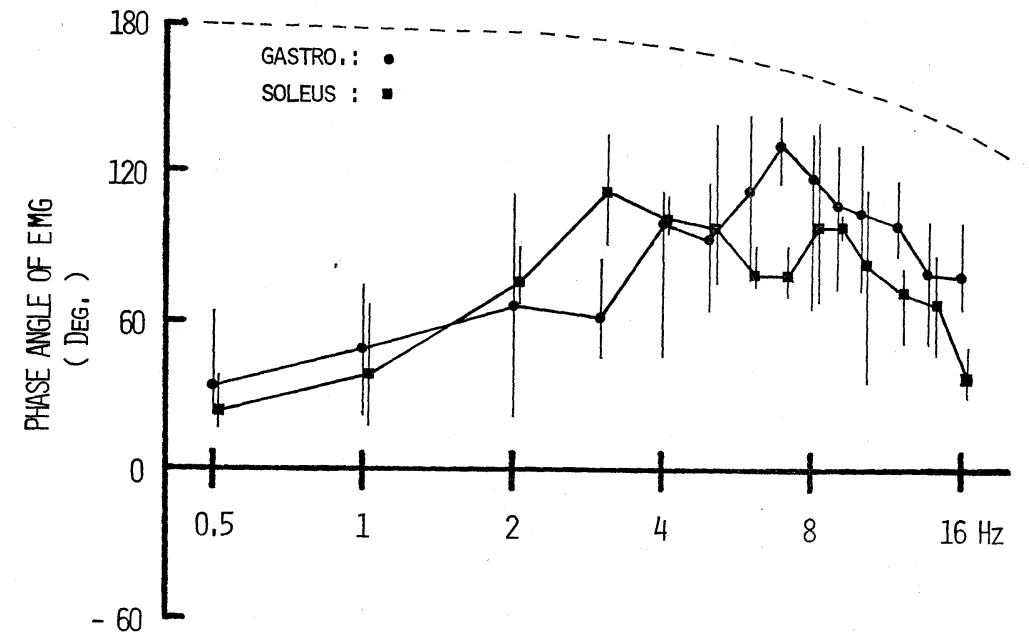

FREQUENCY

Fig. 7. Phase angle of EMG versus frequency during sinusoidal movement ( $2 \mathrm{~mm} \mathrm{p}-\mathrm{p})$ in the gastrocnemius (closed circles) and in the soleus (closed squares) at $8 \mathrm{~mm}$ muscle lengthening. Phase angle of EMG shows average of absolute value calculated from peak of displacement ( 0 degree) with variation among examined preparations (vertical line). Broken line indicates hypothetical position of the eariest EMG which has constant time lag of $8 \mathrm{msec}$ in neural circuit. Phase lead of EMG became larger as frequency increased but began to be small at over $8 \mathrm{~Hz}$ and paralleled the broken line in both muscles. 
of EMG response. It is thought that the EMG produced by the sinusoidal movement took place in the most advanced phase lead at about $8 \mathrm{~Hz}$ and the regression of EMG became inevitable as the time lag in the neural circuit occupied considerable time in each cycle.

\section{Fluctuation of afferent discharges by muscle contraction}

GIa and GIb discharges were recorded simultaneously with the displacement, tension and EMG to investigate mutual relation among these responses. Figure 8 shows the alternate fluctuation of tension and EMG observed at high frequencies. The displacement, tension, EMG and afferent discharges appear from upper trace to bottom in that order and the GIa and GIb were shown by long bar and short bar, respectively. The position of first GIa discharges in the cycle with large tension was compared with that in the cycle with small tension and showed a shift

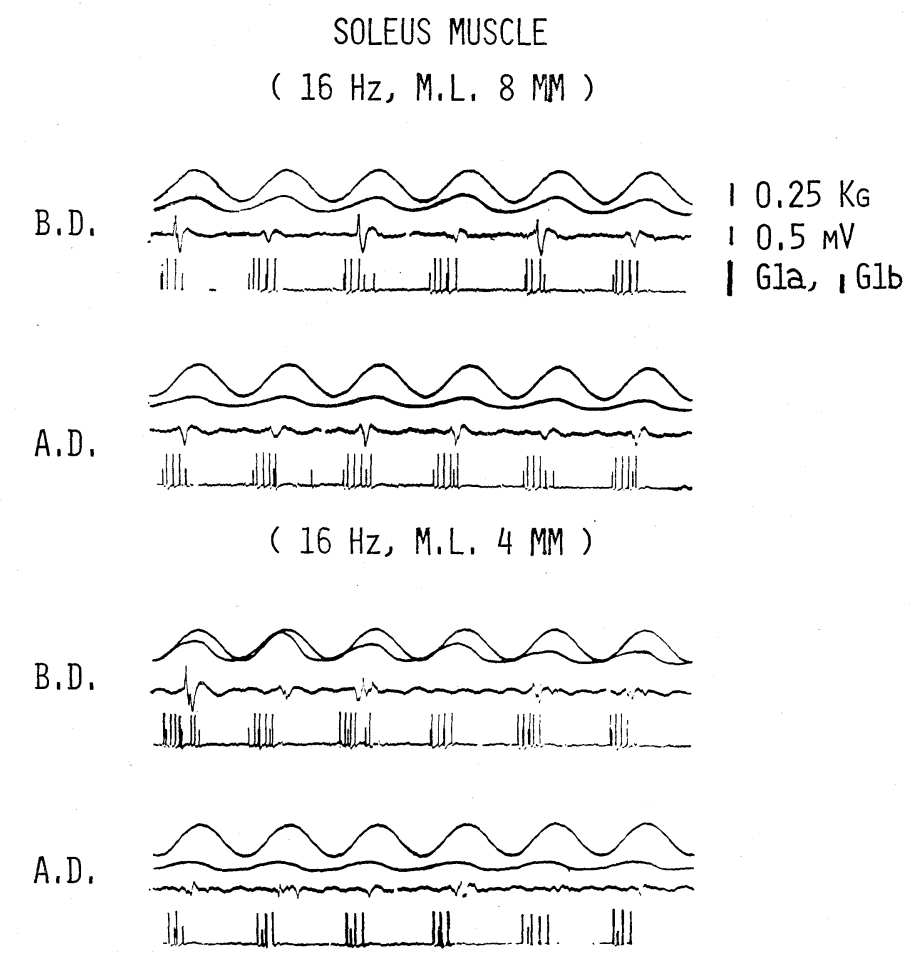

Fig. 8. Simultaneous recording of displacement, tension, EMG, GIa and GIb discharges during sinusoidal movement $(2 \mathrm{~mm} \mathrm{p}$-p) in the soleus at $4 \mathrm{~mm}$ or $8 \mathrm{~mm}$ muscle lengthening. Large bar and small bar in the lowest traces represent retouched GIa and GIb discharges respectively. The second pictures at each lengthening of muscle show the same preparation at $10 \mathrm{~min}$ after Dantrolene sodium injection $(2 \mathrm{mg} / \mathrm{kg}$, i.v.). Note the shift of position of GIb discharges at each cycle. The shift of GIb discharges halted after removing reflex tension by Dantrolene sodium (see text). 
to the left (direction to initiation of stretch) in the cycle with large tension. (See distance between first GIb and first GIa discharge in each cycle of upper pictures during each lengthening of muscle.) On the other hand, the shift of GIa discharges did not resemble the GIb discharges. On a smaller lengthening of muscle, the position of GIa discharges receded in the cycle with large tension. In one preparation (Fig. 8) a response time of the first GIb discharge measured at the dorsal root was $5.1 \mathrm{msec}$ in the cycle with large tension and $7.7 \mathrm{msec}$ in the cycle with small tension. In the cycle where the GIb discharges occurred earlier, the EMG depression was observed despite a large tension. When the muscle

GASTRO, MUSCLE
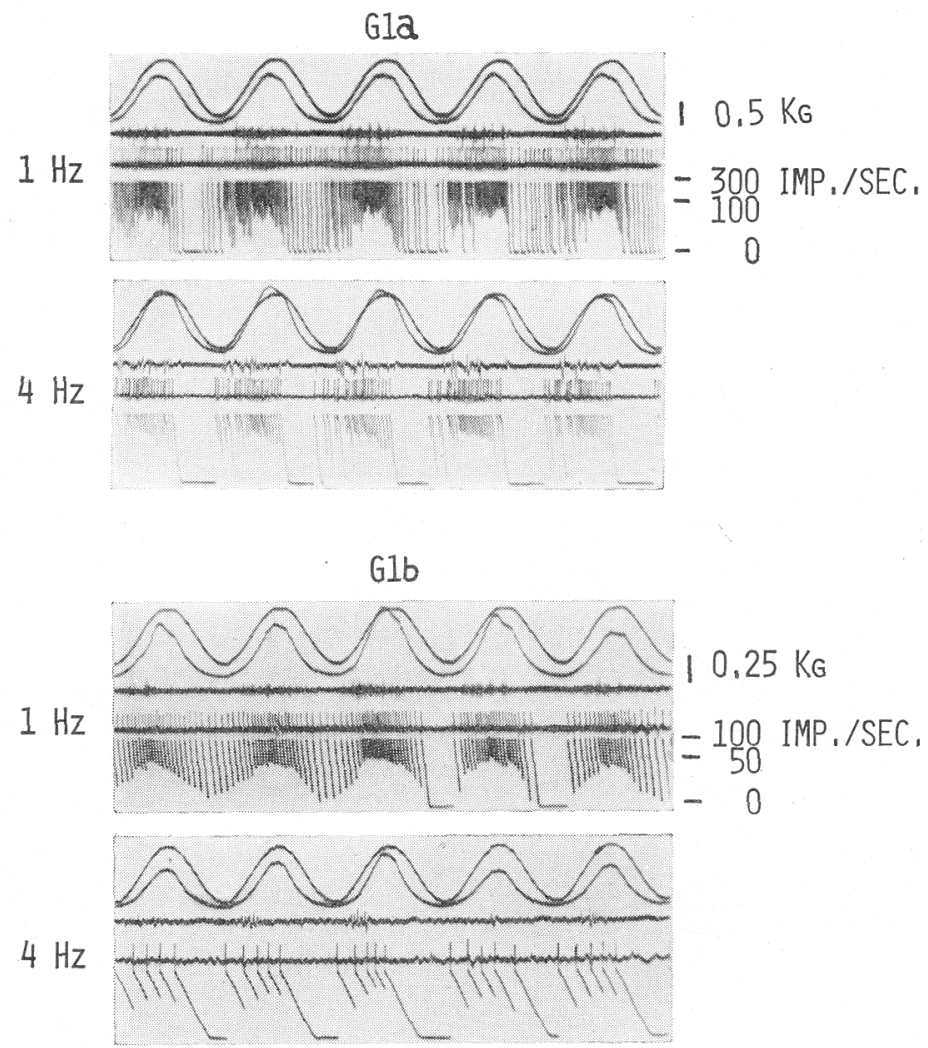

Fig. 9. Simultaneous recording of displacement, tension, EMG and GIa or GIb discharges during sinusoidal movement ( $2 \mathrm{~mm} \mathrm{p}-\mathrm{p})$ in the gastrocnemius at $8 \mathrm{~mm}$ muscle lengthening. Histogram of the lowest trace at each picture shows interspike interval of GIa or GIb discharges. Note the acceleration and pause in GIa discharges (upper two pictures) and acceleration in GIb discharges (lower two pictures) which are related with EMG burst (third trace). 
tension was reduced by Dantrolene sodium* injection $(2 \mathrm{mg} / \mathrm{kg}$, i.v.), the shift of GIb discharges disappeared and the alternate cycle fluctuation of EMG was also less (see lower pictures during each lengthening of muscle). These results may indicate that the alternate cycle inhibition of EMG are caused by the shift of GIb discharges which may effectively inhibit following GIa discharges. A fluctuation of afferent discharges was also observed at low frequencies. As seen in Fig. 9, the GIa discharges increased with the sinusoidal movement but many discontinuance of GIa discharges were observed. The discontinuance of GIa discharges seems to be related to EMG responses; that is, the EMG occurred after an acceleration of few GIa discharges and was seen at the reduction or pause of GIa discharges. The acceleration of GIa discharges started again after a short period and produced the next EMG burst. As frequency of stretching increased, the number of the accelerated GIa discharges and the following EMG burst became few. On the other hand, the GIb discharges increased almost continually with sinusoidal movement but the acceleration of GIb discharges were also seen after EMG burst. These fluctuation of afferent discharges disappeared after cutting the ventral roots.

The acceleration and pause of GIa discharges may explain the three types of EMG patterns: continuous group discharge, two or three group discharges with intermittance, and one spikelike discharge (see white dots in Fig. 1). The continuous group discharges were seen at low frequencies $(0.5$ to $2 \mathrm{~Hz})$ and formed a rhombic or spindle shape. The intermittent group discharges began to appear at middle frequencies ( 4 to $8 \mathrm{~Hz}$ ) and its average intermittance was about $10 \mathrm{msec}$. At high frequencies (over $12 \mathrm{~Hz}$ ), the intermittent group discharges converged into one spikelike discharge. Similar alteration of EMG patterns was described in the soleus by JANSEN and RACK (1966) as continuous, vigorous and short bursts.

\section{DISCUSSION}

The responses of tension and EMG during sinusoidal movement in the soleus showed results similar to those described by JANSEN and RACK (1966). Comparison of responses between gastrocnemius and soleus call attention to the fact that the tension decay, phase lag of tension, and alternate cycle fluctuation of tension and EMG occurred at higher frequencies in the gastrocnemius than in the soleus. The mutual relations of these phenomena are next discussed with concern given to time lags of the conduction time of reflex arc and the contraction times of the muscles.

* Dantrolene sodium, (1-[5-( $p$-nitrophenyl)flufurylidine amino] hydantoin sodium hydrate), synthesized by Norwich Pharmacal Company (SNYDER et al., 1967) produces relaxation of skeletal muscle at a peripheral site rather than at a central nervous system site (HoNKop et al., 1970; Heald and Matsumoto, 1971). It is also reported that neuromuscular transmission is not affected by the drug in frog muscle (Ellis and CARPENTER, 1971; ZoRYCHTA et al., 1971). 
The amplitude of tension was increased or maintained up to $9 \mathrm{~Hz}$ in the gastrocnemius and up to $4 \mathrm{~Hz}$ in the soleus but decreased after these frequencies, despite the amplitude of EMG which increased with increasing frequencies. As frequency increased, the development of tension may be produced by the increase of EMG amplitude and the earlier appearance of EMG phase angle as described in the soleus by JANSEN and RACK (1966). The phase angle of EMG at these frequencies corresponds to the most rapid stretching period of sinusoidal movement. The relation is, however, disrupted by the receding of EMG phase angle at over $8 \mathrm{~Hz}$ in the gastrocnemius and at over $4 \mathrm{~Hz}$ in the soleus. The tension will be rather small at over these frequencies at which the phase angle of EMG retards as the sinusoidal movement fails to affect the greatest activity of these muscles. The receding of EMG phase angle is inevitable at high frequencies at over $8 \mathrm{~Hz}$ in both muscles since the time lag of the conduction time of reflex arc occupies considerable time of each cycle.

The phase lag of tension occurred at over $12 \mathrm{~Hz}$ in the gastrocnemius and at over $6 \mathrm{~Hz}$ in the soleus. The phase lag observed at different frequency in these muscles also seems to be caused by the receding of EMG phase angle. The phase lead in the gastrocnemius and the phase lag in the soleus at around 8 to $10 \mathrm{~Hz}$, however, are not explained by the retreat of EMG phase angle, because the phase angle in both muscles show the almost same value at these frequencies. It is, therefore, supposed that the difference of damping properties in these two muscles may cause the phase lag at different frequencies.

Different time courses of the contraction in these muscles are noticed as the lengthening time of the sinusoidal movement at the frequencies corresponds closely to the isometric contraction time of these two muscles. When the reflex arc conduction time is $8 \mathrm{msec}$ and the contraction time is $30 \mathrm{msec}$ in the gastrocnemius and $90 \mathrm{msec}$ in the soleus, the peak of isometric tension caused by tendon jerk, for example, arises at $38 \mathrm{msec}$ and $98 \mathrm{msec}$ later from the time of the tendon hit, respectively. (These times were assumed from authors' data.) The frequency, which has the lengthening time 'of 38 and $98 \mathrm{msec}$, corresponds to 13.2 and $5.1 \mathrm{~Hz}$, respectively. The peak of isometric tension in these muscles caused by EMG which has the time lag of $8 \mathrm{msec}$ consistent with the peak of sinusoidal movement at $13.2 \mathrm{~Hz}$ in the gastrocnemius and at $5.1 \mathrm{~Hz}$ in the soleus (Fig. 10). Large open symbols show hypothetical position of peak isometric tension in the gastrocnemius (circles) and in the soleus (squares) produced by EMG spike which arises just $8 \mathrm{msec}$ later from the beginning of sinusoidal movement. (The position of the EMG corresponds to the broken line in Fig. 7.) Small open circles and squares also show hypothetical position of peak isometric tension calculated from the actual phase angle of EMG in Fig. 7. Small solid symbols represent the actual phase angle of reflex tension (the same as in Fig. 5 but plotted on a different scale).

Both hypothetical phase angles of the tension suggest the occurrence of the phase lag at around $14 \mathrm{~Hz}$ in the gastrocnemius and at around $6 \mathrm{~Hz}$ in the soleus. 


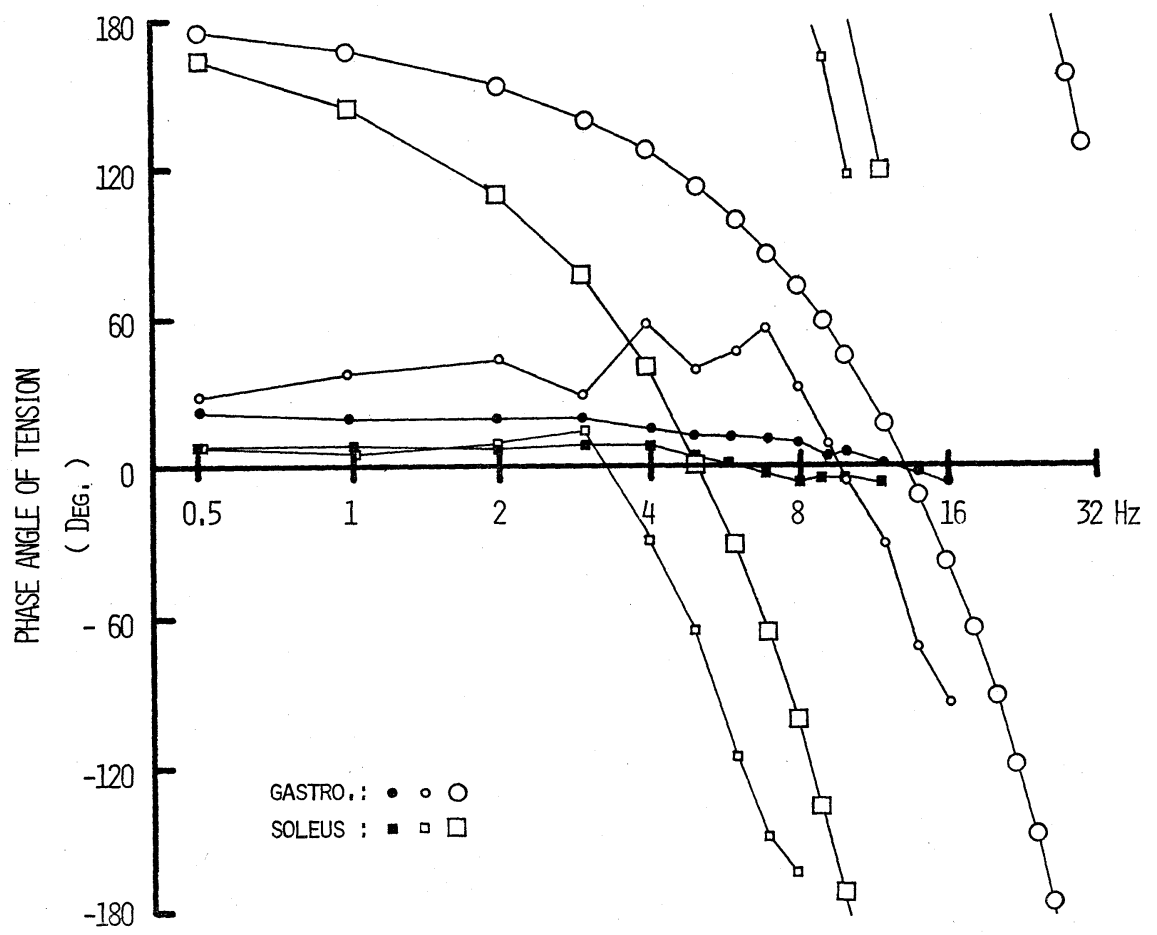

FREQUENCY

Fig. 10. Mutual relation between phase angle of tension and time lag of the contraction time. Large and small open symbols show hypothetical position of peak in isometric tension calculated from position of the earliest EMG (broken line in Fig. 7) and actual phase angle of EMG (Fig. 7) respectively. Contraction time was used $30 \mathrm{msec}$ in the gastrocnemius (circles) and $90 \mathrm{msec}$ in the soleus (squares). 0 degree line corresponds to position of maximum sinusoidal movement and 180 degrees line indicates initiation of the movement. Note that peak of isometric tension rises after maximum displacement at over $14 \mathrm{~Hz}$ in the gastrocnemius and at over $6 \mathrm{~Hz}$ in the soleus even if EMG occurred at the eariest period of sinusoidal movement and that isometric tension begins to affect next cycle at over $26 \mathrm{~Hz}$ in the gastrocnemius and at over $10 \mathrm{~Hz}$ in the soleus.

The actual values of the phase lead and the phase lag are, however, smaller and closer to zero degree than the ones calculated from the actual phase angle of EMG. It may be the reason the actual EMG discharges are not completely synchronous as is the hypothetical one and that the damping properties of these muscles act on the phase angle to keep it around zero level during sinusoidal movement (JANSEN and RACK, 1966; HERMAN et al., 1973). That is, when the active muscle is stretched, the tension increased with the stretch and rapidly decays as soon as the muscle begins to be shorten (RACK, 1966; JOYCE and RACK, 1969; JOYCE et al., 1969). The velocity of lengthening may, therefore, increase the muscle stiffness when the muscle is stretched during contraction and the tension 
may develop until the balance between the state of the active muscle and the effect of the velocity of lengthening collapses. During the muscle relaxation, the velocity of the shortening may act on the stiffness of the muscle by reverse relation.

The different effects due to the different time courses of the contraction in these two muscles were also seen in the responses of the reflex tension. The tension produced by the first cycle began to affect the second cycle at 12 to $16 \mathrm{~Hz}$ in the gastrocnemius and at 7 to $8 \mathrm{~Hz}$ in the soleus. But the combination between the active muscle and the velocity of displacement is not suitable to produce a large tension and a small fluctuation of the tension is observed as seen in Figs. 1 and 2 (see arrows). According to the hypothetical phase angle of the tension in Fig. 10, a suitable frequency is expected at around $26 \mathrm{~Hz}$ in the gastrocnemius and at around $10 \mathrm{~Hz}$ in the soleus. In fact, one of the preparations represented in Fig. 2 shows a relatively large tension in the second cycle as compared with the first cycle at $24 \mathrm{~Hz}$ in the gastrocnemius and at $14 \mathrm{~Hz}$ in the soleus. Thus, the effect of tension on the next cycle will be observed earlier in the soleus than in the gastrocnemius as the time course of the contraction is longer in the soleus than in the gastrocnemius.

Tension produced by stretch reflex itself is one of the input to afferent receptors and seems to cause the deformation, discontinuation or trembling, of the afferent discharges (Fig. 9) which may result in discontinuation of EMG. At high frequencies, the effect of own reflex tension to the receptors are pronounced and seems to cause the alternate cycle fluctuation of EMG at 14 to $16 \mathrm{~Hz}$ in the soleus and at 22 to $24 \mathrm{~Hz}$ in the gastrocnemius. (EMG depression at around $16 \mathrm{~Hz}$ in the gastrocnemius may be related to the lack of motoneuron discharge described by Homma et al., 1970.) The reflex tension seems to force the inhibitory effect of GIb discharges to the motoneuron by not only increasing the frequency but also by leading the phase lead of discharges at high frequencies. The retreat of EMG phase angle at around $6 \mathrm{~Hz}$ in the soleus may be caused by the reason mentioned above as well as an unloading effect on muscle spindles as discussed by JANSEN and RACK (1966). The GIb discharge affected by own muscle contraction are supposed to play considerable effect to the stretch reflex system at high frequencies as well as the suggestion that the reflex influence of GIb discharge become relatively stronger at high frequencies (STUART et al., 1965).

The deformed GIa discharges affected by own muscle contraction seem to suggest recurrent effects of own reflex tension in the reflex system. The effect may, for example, contribute to an avoidance of fatigue of the motoneuron and muscle, a rejection of receptors and a dependency or allowance of other input to the same motoneurons etc. Further, it is noticed that the frequency of GIa discharge after pause was very high. The phasic burst after pause of GIa discharges, which are observed at an isometric twitch contraction, may take part in the increase of the discharge. The discontinuous, accelerated discharge of GIa may contribute to keep a level of an activity of motoneuron at low frequency input or 
static stretch. In other words, when the muscle tension is removed, the activity of the motoneuron may be reduced as well as the deterioration of the muscle tension itself. This speculation may support clinical data on spastic patients (HERMAN et al., 1972).

These results confirm that the time lags of peripheral nerve and the contraction times of muscles are two of mean possible factors for the limitation of reflex responses, and that the contraction of muscle itself recurrently affected the input flow of afferent discharges to the spinal. Further, the contractile properties of the muscle seems to control the amplitude and the phase angle of tension, especially latter, during sinusoidal movement as well as a control by cerebellum as described by Higgins and Glaser (1964). The mutual relationship of reflex tension and EMG between preceding cycle and following cycles indicates that any events produced by the previous cyisles are important conditions to the next cycles. It is therefore necessary to pay attention to the selection of frequency to be used for experiments and analysis of data unless the effect of the preceding cycle is to be considered as another input to the following events.

The authors are grateful to Mrs. Babara Cozzen for her calculation of data, to Mr. Fred Kugler for his technical assistance and to Mr. James Vorges who cared for the cats prior to experiments. This study was supported by grants No. 16 p 56804/3-09 from the Department of Health, Education, and Welfare, the Social and Rehabilitative Servics, Washington, D.C., U.S.A.

\section{REFERENCES}

Ellis, K. O. and CARPENTER, J. F. (1971) The effect of Dantrolene sodium (F-440) on skeletal muscle. Fed. Proc., 30: 670.

Gottlieb, G. L., Agarwal, G. C., and Stark, L. (1970) Interactions between voluntary and postural mechanism of the human motor system. J. Neurophysiol., 33: 365-381.

GranIT, R. (1958) Neuromuscular interaction in postural tone of the cat's isometric soleus muscle. J. Physiol., 143: 387-402.

Heald, D. E. and Matsumoto, Y. (1971) Inhibition of contraction of frog skeletal muscle by Dantrolene sodium. Fed. Proc., 30: 378.

Herman, R., Freedman, W., Monster, A. V., and Tamai, Y. (1973) A systematic analysis of myotatic reflex activity in human spastic muscle. In New Developments in Electromyography and Clinical Neurophysiology, ed by Desmedt, J. E. S. Karger, Basel, pp. 556-578.

Herman, R., MAYeR, N., and MCComber, S. A. (1972) Clinical pharmacophysiology of Dantrolene sodium. Am. J. Phys. Med., 51: 296-311.

Higgins, D. C. and Glaser, G. H. (1964) Stretch responses during chronic cerebellar ablation. A study of reflex instability. J. Neurophysiol., 27: 49-62.

Higgins, D. C., Partridge, L. D., and Glaser, G. H. (1962) A transient cerebellar influence on stretch responses. J. Neurophysiol., 25: 684-692.

Homma, S., Ishikawa, K., and StuarT, D. G. (1970) Motoneuron responses to linearly rising muscle stretch. Am. J. Phys. Med., 49: 290-306.

Honkop, L. J., Halliday, R. P., and Wessels, F. L. (1970) Dantrolene, 1-[5-( $p$-nitrophenyl)furfurylidene amino] hydantoin, a unique skeletal muscle relaxant. Pharmacologist, 12: 301.

JANSEN, J. K. S. and RACK, P. M. H. (1966) The reflex response to sinusoidal stretching of 
soleus in the decerebrate cat. J. Physiol., 183: 15-36.

JoyCE, G. C. and RACK, P. M. H. (1969) Isotonic lengthening and shortening movement of cat soleus muscle. J. Physiol., 204: 475-491.

Joyce, G. C., Rack, P. M. H., and Westbury, D. R. (1969) The mechanical properties of cat soleus muscle during controlled lengthening and shortening movement. J. Physiol., 204: 461-474.

Liddell, E. G. T. and Scherrington, C. S. (1924) Reflexes in response to stretch (Myotatic reflexes). Proc. Roy. Soc. B, 96: 212-242.

Lippold, O. C. J., Redfearn, J. W. T., and Vučo, J. (1958) The effect of sinusoidal stretching upon the activity of stretch receptors in voluntary muscle and their reflex responses. $J$. Physiol., 144: 373-386.

Matthews, P. B. C. (1959a) The dependence of tension upon extension in the stretch reflex of the soleus muscle of the decerebrate cat. J. Physiol., 147: 521-546.

Matthews, P.B. C. (1959b) A study of certain factors influencing the stretch reflex of the decerebrate cat. J. Physiol., 147: 547-564.

Partridge, L. D. and Glaser, G. H. (1960) Adaptation in regulation of movement and posture. A study of stretch responses in spastic animals. J. Neurophysiol., 23: 257-268.

Poppele, R. E. and Terzuolo, C. A. (1968) Myotatic reflex: Its input-output relation. Science, 159: 743-745.

RACK, P. M.H. (1966) The behaviour of a mammalian muscle during sinusoidal stretching. J. Physiol., 183: 1-14.

Roberts, T. D. M. (1963) Rhythmic excitation of stretch reflex, revealing (a) hysteresis and (b) a difference between responses to pulling and to stretching. Quart. J. Exp. Physiol., 48: 328-345.

Rosenthal, N. P., McKean, T. A., Roberts, W. J., and Terzuolo, C. A. (1970) Frequency analysis of stretch reflex and its main subsystems in triceps surae muscles of the cat. $J$. Neurophysiol., 33: 713-749.

SNYder, H. R., Jr., DAvis, C. S., Bickerton, R. K., and HAlliday, R. P. (1967) 1-[(5-Arylfurfurylidene)amino]hydantoins. A new class of muscle relaxants. J. Med. Chem., 10: 807-810.

Stuart, D., OtT, K., IshiKawa, K., and Eldred, E. (1965) Muscle receptor responses to sinusoidal stretch. Exp. Neurology, 13: 82-95.

Zorychta, E., Esplin, D. W., CAPek, R., and Lastrowecka, A. (1971) The action of Dantrolene on extrafusal and intrafusal striated muscle. Fed. Proc., 30: 669. 